\title{
New variables for gyrokinetic electromagnetic simulations
}

\author{
Alexey Mishchenko* \\ Max Planck Institute for Plasma Physics, D-17491 Greifswald, Germany \\ Michael Cole, Ralf Kleiber, and Axel Könies \\ Max Planck Institute for Plasma Physics, D-17491 Greifswald, Germany
}

(Dated: May 8, 2014)

\begin{abstract}
A new approach to electromagnetic gyrokinetic simulations based on modified gyrokinetic theory is described. The method is validated using a particle-in-cell code. The Toroidal Alfvén Eigenmode at low perpendicular mode numbers, the so-called "MHD limit", has been successfully simulated using this method.
\end{abstract}

* alexey.mishchenko@ipp.mpg.de 


\section{INTRODUCTION}

There is a large number of cases in fusion plasmas in which electromagnetic kinetic effects are of importance. These include Alfvénic dynamics, tearing modes, electromagnetic drift microinstabilities etc. All these phenomena have characteristic frequencies smaller than the gyrofrequency. This permits the gyrokinetic theory to be applied for them, with a substantial reduction of the computational cost as a potential consequence. Unfortunately, gyrokinetic electromagnetic simulations suffer from the so-called cancellation problem [1]. The problem has been addressed within the particle-in-cell framework in Refs. [2-5] and using the Eulerian approach in Ref. [6]. Progress in understanding numerical issues underlying the electromagnetic gyrokinetic scheme has made it possible to simulate globally Alfvénic modes, such as the Toroidal Alfvén Eigenmodes (TAE) [7-9], Global Alfvén Eigenmodes [7], internal $m=1$ kink mode and $m=1$ reconnecting modes [10]. Despite the progress achieved, the solution of the cancellation problem discussed in Refs. $[2,5,6]$ remains incomplete. Numerical problems are still observed with many gyrokinetic codes, in particular when attempting global electromagnetic simulations of low- $\mathbf{k}_{\perp}$ modes (the Magnetohydrodynamical, MHD, limit) in realistic toroidal geometry. Presently, local (flux-tube), truncated (fluid-electron [11, 12]) or hybrid (kinetic MHD) schemes remain the method of choice in many cases when simulating electromagnetic physics in realistic devices. It is, however, also desirable to assess the electron kinetics, such as trapped-electron effects, or non-adiabatic electron dynamics at resonant flux surfaces.

In this paper, we present a novel approach to the solution of the cancellation problem based on a modification of the gyrokinetic theory [13]. The gyrokinetic variables and equations are generated by an explicit form of parallel Ohm's law anticipated as a good approximation for the parallel electric field. We verify our approach using the global gyrokinetic particle-in-cell code EUTERPE [14]. The example of a TAE mode at low perpendicular mode numbers is considered. It is found that our method greatly facilitates the global gyrokinetic simulations of MHD phenomena.

The paper is organised as follows. In Sec. II, our method is introduced. The simulations are described in Sec. III. Finally, we make our conclusions in Sec. IV. 


\section{THEORY}

Following Ref. [15], we start our derivation from the Poincaré-Cartan form:

$$
\gamma=q \mathbf{A}^{*} \cdot \mathrm{d} \mathbf{R}+\frac{m}{q} \mu \mathrm{d} \theta+q A_{\|} \mathbf{b} \cdot \mathrm{d} \mathbf{x}-\left[\frac{m v_{\|}^{2}}{2}+\mu B+q \phi\right] \mathrm{d} t
$$

Here, $\mathbf{R}$ is the guiding-centre coordinate, $\mathbf{x}=\mathbf{R}+\boldsymbol{\rho}$ with $\boldsymbol{\rho}(\theta)$ being the gyroradius, $\theta$ is the gyro-phase (the fast variable), $\mathbf{A}^{*}=\mathbf{A}+\left(m v_{\|} / q\right) \mathbf{b}$ the so-called modified vector potential, A the magnetic potential corresponding to the equilibrium magnetic field $\mathbf{B}=\nabla \times \mathbf{A}$ and $\mathbf{b}=\mathbf{B} / B$ the unit vector in the direction of the equilibrium magnetic field. The gyrokinetic theory can be derived applying the Lie transform technique to the Poincaré-Cartan form along with the requirement of "gyro-independence" of the final result (i. e. requiring the Lie-transformed dynamics to have no explicit dependence on the gyro-phase $\theta$, see Ref. [15] for details). The gyro-dependence enters Eq. (1) only through the perturbed fields: the electrostatic potential $\phi(\mathbf{x})$ and the parallel magnetic potential $A_{\|}(\mathbf{x})$.

There are two approaches to the gyrokinetic theory described in literature (see the discussion in Ref. [1]). In the so-called $p_{\|}$-formulation (also called the "hamiltonian model" in Ref. [15]), one shifts both the electrostatic and magnetic field perturbations into the gyrokinetic Hamiltonian. The gyrokinetic Poincaré-Cartan form is then as follows, only the zeroand the first-order contributions are kept for simplicity in the expression below:

$$
\Gamma=q \mathbf{A}^{*} \cdot \mathrm{d} \mathbf{R}+\frac{m}{q} \mu \mathrm{d} \theta-\left[\frac{m v_{\|}^{2}}{2}+\mu B+q\left\langle\phi-v_{\|} A_{\|}\right\rangle\right] \mathrm{d} t
$$

Here $\langle\ldots\rangle$ denotes the gyro-average defined as usual. In the $p_{\|}$-formulation, the Poisson brackets of the gyrokinetic theory coincide with the "unperturbed" drift-kinetic Poisson brackets [15], but the cancellation problem appears in Ampere's law, making the electromagnetic simulations cumbersome. Note that all the phase-space variables are given by series in perturbed fields $\phi$ and $A_{\|}$(see Ref. [15] for details).

Alternatively, in the $v_{\|}$-formulation (also called the "symplectic model" [15]), one keeps the structure of the gyrokinetic Poincaré-Cartan form similar to the original, as in Eq. (1). Then, the gyrokinetic Poincaré-Cartan form shall be written as below, where, for simplicity, only the low-order contributions are kept:

$$
\Gamma=q \mathbf{A}^{*} \cdot \mathrm{d} \mathbf{R}+\frac{m}{q} \mu \mathrm{d} \theta+q\left\langle A_{\|}\right\rangle \mathbf{b} \cdot \mathrm{d} \mathbf{R}-\left[\frac{m v_{\|}^{2}}{2}+\mu B+q\langle\phi\rangle\right] \mathrm{d} t
$$


In the $v_{\|}$-formulation, the parallel Ampere's law does not have the cancellation problem but the Poisson brackets are "perturbed". As a consequence, terms proportional to $\partial\left\langle A_{\|}\right\rangle / \partial t$ appear in the equations of motion. This can also be a problem for simulations (see Ref. [1]). Note that here again the phase-space variables are given by series in perturbed fields, but the explicit form of these series differs from the $p_{\|}$-formulation. In this sense, one should distinguish between e.g. the symplectic parallel velocity variable and the hamiltonian one.

In our approach, we employ a mixture of the $p_{\|^{-}}$and $v_{\|}$-formulations which is generated by a particular, appropriately chosen, version of parallel Ohm's law, the ideal version being used throughout this paper. For that sake, we "add a zero" to the original Poincaré-Cartan form Eq. (1), rewriting it as follows:

$$
\begin{aligned}
\gamma= & q \mathbf{A}^{*} \cdot \mathrm{d} \mathbf{R}+\frac{m}{q} \mu \mathrm{d} \theta+q A_{\|} \mathbf{b} \cdot \mathrm{d} \mathbf{x}+ \\
& +\left[q A_{\|}^{(\mathrm{MHD})} \mathbf{b} \cdot \mathrm{d} \mathbf{x}-q A_{\|}^{(\mathrm{MHD})} \mathbf{b} \cdot \mathrm{d} \mathbf{x}\right]-\left[\frac{m v_{\|}^{2}}{2}+\mu B+q \phi\right] \mathrm{d} t
\end{aligned}
$$

The auxiliary quantity $A_{\|}^{(\mathrm{MHD})}$ added here is chosen to obey Ohm's law:

$$
\frac{\partial}{\partial t} A_{\|}^{(\mathrm{MHD})}+\mathbf{b} \cdot \nabla \phi=0
$$

Introducing the notation $\delta A_{\|}=A_{\|}-A_{\|}^{(\mathrm{MHD})}$, we obtain the following Poincaré-Cartan form as a starting point for the appropriate gyrokinetic theory:

$$
\gamma=q \mathbf{A}^{*} \cdot \mathrm{d} \mathbf{R}+\frac{m}{q} \mu \mathrm{d} \theta+q A_{\|}^{(\mathrm{MHD})} \mathbf{b} \cdot \mathrm{d} \mathbf{x}+q \delta A_{\|} \mathbf{b} \cdot \mathrm{d} \mathbf{x}-\left[\frac{m v_{\|}^{2}}{2}+\mu B+q \phi\right] \mathrm{d} t
$$

Note that $A_{\|}^{(\mathrm{MHD})}$ represents a good approximation of the parallel magnetic potential for MHD-like modes. In other words, the relations $\delta A_{\|} \ll A_{\|}$and $A_{\|} \sim A_{\|}^{(\mathrm{MHD})}$ are expected to be satisfied in the regime with $E_{\|}=\partial A_{\|} / \partial t+\nabla_{\|} \phi \approx 0$. In our derivation we employ the " $v_{\|}$-formulation" (symplectic transformation [15]) for $A_{\|}^{(\mathrm{MHD})}$ and the " $p_{\|}$-formulation" (hamiltonian transformation [15]) for $\delta A_{\|}$. As a consequence, we would obtain the terms proportional to $\partial\left\langle A_{\|}^{(\mathrm{MHD})}\right\rangle / \partial t$ in the gyrocenter equations of motion. But such terms would not be a problem since $\partial\left\langle A_{\|}^{(\mathrm{MHD})}\right\rangle / \partial t$ is explicitly known from Ohm's law. Also, the cancellation problem will still appear, as is the case in the usual $p_{\|}$-formulation, but now the terms to be cancelled are proportional to $\delta A_{\|}$. This should make the cancellation problem much weaker, at least in the regimes with $\delta A_{\|} \ll A_{\|}^{(\mathrm{MHD})}$. In other words, $A_{\|}^{(\mathrm{MHD})}$ should be a good "physical" guess for the actual magnetic potential for the scheme to work. The 
resulting gyrokinetic Poincaré-Cartan form in the 'mixed-variable' formulation, generated by the Ohm's law, is to first order

$$
\Gamma=q \mathbf{A}^{*} \cdot \mathrm{d} \mathbf{R}+\frac{m}{q} \mu \mathrm{d} \theta+q\left\langle A_{\|}^{(\mathrm{MHD})}\right\rangle \mathbf{b} \cdot \mathrm{d} \mathbf{R}-\left[\frac{m v_{\|}^{2}}{2}+\mu B+q\left\langle\phi-v_{\|} \delta A_{\|}\right\rangle\right] \mathrm{d} t
$$

As has been mentioned above, the phase-space variables are given by series in the perturbed fields whose precise form depend on the formulation employed. Proceeding further in the standard way [15], we obtain the gyrocenter equations of motion.

$$
\begin{aligned}
& \dot{\mathbf{R}}^{(1)}=\frac{\mathbf{b}}{B_{\|}^{*}} \times \nabla\left\langle\phi-v_{\|} A_{\|}^{(\mathrm{MHD})}-v_{\|} \delta A_{\|}\right\rangle-\frac{q}{m}\left\langle\delta A_{\|}\right\rangle \mathbf{b}^{*} \\
& \dot{v}_{\|}^{(1)}=-\left[\frac{v_{\|}}{B_{\|}^{*}}(\nabla \times \mathbf{b}) \cdot \nabla \phi-\frac{q}{m} v_{\|} \mathbf{b}^{*} \cdot \nabla\left\langle\delta A_{\|}\right\rangle+\frac{\mu}{m} \frac{\mathbf{b} \times \nabla B}{B_{\|}^{*}} \cdot \nabla\left\langle A_{\|}^{(\mathrm{MHD})}\right\rangle\right]
\end{aligned}
$$

The zeroth-order gyrocenter characteristics are as usual:

$$
\dot{\mathbf{R}}^{(0)}=v_{\|} \mathbf{b}^{*}+\frac{1}{q B_{\|}^{*}} \mathbf{b} \times \mu \nabla B, \quad \dot{v}_{\|}^{(0)}=-\frac{\mu}{m} \mathbf{b}^{*} \cdot \nabla B
$$

Here, we use the standard notations $B_{\|}^{*}=\mathbf{b} \cdot \nabla \times \mathbf{A}^{*}$ and $\mathbf{b}^{*}=\nabla \times \mathbf{A}^{*} / B_{\|}^{*}$.

The pullback of the distribution function in the "mixed variables" can be obtained in the usual way following Ref. [15]:

$$
f_{1}=\bar{f}_{1}+\frac{e}{B}\left(\widetilde{\phi}-v_{\|} \delta \widetilde{A}_{\|}-v_{\|} \widetilde{A}_{\|}^{(\mathrm{MHD})}\right) \frac{\partial F_{0}}{\partial \mu}+\frac{e}{m}\left(\delta A_{\|}+\widetilde{A}_{\|}^{(\mathrm{MHD})}\right) \frac{\partial F_{0}}{\partial v_{\|}}
$$

Here, the notation $\widetilde{Q}=Q-\langle Q\rangle$ is used for the gyro-dependent part of a quantity $Q$ (which stands for the perturbed fields), $F_{0}$ is the ambient distribution function (a Maxwellian throughout this paper), $\bar{f}_{1}$ is the gyrokinetic distribution function satisfying the Vlasov equation with the characteristics given by Eqs. (8)-(10) and $f_{1}$ is the "guiding-center Vlasov distribution" [15]. Substituting the pullback Eq. (11) into the quasineutrality equation and parallel Ampere's law:

$$
\begin{aligned}
& n_{1 i}-n_{1 e}=0, \quad-\nabla_{\perp}^{2} A_{\|}=\mu_{0}\left(j_{\| 1 i}+j_{\| 1 e}\right) \\
& n_{1 s}=\int \mathrm{d}^{6} Z f_{1 s} \delta(\mathbf{R}+\boldsymbol{\rho}-\mathbf{x}), \quad j_{\| 1 s}=q_{s} \int \mathrm{d}^{6} Z f_{1 s} v_{\|} \delta(\mathbf{R}+\boldsymbol{\rho}-\mathbf{x}),
\end{aligned}
$$

one can reformulate these equations in the "mixed variables" as follows:

$$
\begin{aligned}
& \int \frac{q_{i} F_{0 i}}{T_{i}}(\phi-\langle\phi\rangle) \delta(\mathbf{R}+\boldsymbol{\rho}-\mathbf{x}) \mathrm{d}^{6} Z=\bar{n}_{1 i}-\bar{n}_{1 e} \\
& \left(\frac{\beta_{i}}{\rho_{i}^{2}}+\frac{\beta_{e}}{\rho_{e}^{2}}-\nabla_{\perp}^{2}\right) \delta A_{\|}-\nabla_{\perp}^{2} A_{\|}^{(\mathrm{MHD})}=\mu_{0}\left(\bar{j}_{\| 1 i}+\bar{j}_{\| 1 e}\right)
\end{aligned}
$$


with the usual notations: the mixed-variable gyrocenter density $\bar{n}_{1 s}=\int \mathrm{d}^{6} Z \bar{f}_{1 s} \delta(\mathbf{R}+\boldsymbol{\rho}-$ $\mathbf{x}$ ), whose relation to the physical density depends on the particular formulation of the gyrokinetic theory used; the mixed-variable gyrocenter current $\bar{j}_{\| 1 s}=q_{s} \int \mathrm{d}^{6} Z \bar{f}_{1 s} v_{\|} \delta(\mathbf{R}+$ $\boldsymbol{\rho}-\mathbf{x})$, related to the physical current by the pullback; the particle charge $q_{s}$; the gyrokinetic phase-space volume $\mathrm{d}^{6} Z=B_{\|}^{*} \mathrm{~d} \mathbf{R} \mathrm{d} v_{\|} \mathrm{d} \mu \mathrm{d} \theta$; the thermal gyroradius $\rho_{s}=\sqrt{m_{s} T_{s}} /(e B)$; and the plasma beta corresponding to a particular species $\beta_{s}=\mu_{0} n_{0} T_{s} / B_{0}^{2}$. As has been mentioned, the cancellation problem, explicitly appearing in Eq. (15), is now much weaker than it is in the $p_{\|}$-formulation, particularly in cases when $\delta A_{\|} \ll A_{\|}$. In this paper, we have introduced the mixed-variable equations using the Lie transform technique. In the appendix, we discuss briefly the direct variable transformation approach. A complete derivation using the direct method is beyond the scope of the present paper and can be considered in future.

\section{SIMULATIONS}

In this section, we employ the gyrokinetic particle-in-cell (PIC) code EUTERPE [14] to demonstrate that the approach described in Sec. II indeed works for MHD-like modes.

EUTERPE is an extension of the GYGLES code [7-10] which permits nonlinear and nonaxisymmetric simulations (not undertaken in this paper). The code solves the gyrokinetic equation using the characteristics Eqs. (8-10). The perturbed fields $\phi, \delta A_{\|}$and $A_{\|}^{(\mathrm{MHD})}$ are found numerically solving the quasineutrality equation (14), parallel Ampère's law, Eq. (15), and parallel Ohm's law, Eq. (5). Here, the first two equations (14) and (15) represent boundary-value problems whereas the last one, Eq. (5), is an initial value-problem. We choose $A_{\|}^{(\mathrm{MHD})}(t=0)=0$ as the initial condition for Ohm's law.

The perturbed part of the distribution function is discretized in EUTERPE with markers:

$$
\delta f_{s}\left(\mathbf{R}, v_{\|}, \mu, t\right)=\sum_{\nu=1}^{N_{p}} w_{s \nu}(t) \delta\left(\mathbf{R}-\mathbf{R}_{\nu}\right) \delta\left(v_{\|}-v_{\nu \|}\right) \delta\left(\mu-\mu_{\nu}\right),
$$

where $N_{p}$ is the number of markers, $\left(\mathbf{R}_{\nu}, v_{\nu \|}, \mu_{\nu}\right)$ are the marker phase space coordinates and $w_{s \nu}$ is the weight of a marker. The electrostatic and magnetic potentials are discretized with the finite-element method (Ritz-Galerkin scheme):

$$
\phi(\mathbf{x})=\sum_{l=1}^{N_{s}} \phi_{l} \Lambda_{l}(\mathbf{x}), \quad A_{\|}(\mathbf{x})=\sum_{l=1}^{N_{s}} a_{l} \Lambda_{l}(\mathbf{x})
$$


where $\Lambda_{l}(\mathbf{x})$ are the finite elements (tensor product of B splines $\left.[16,17]\right), N_{s}$ is the total number of the finite elements, $\phi_{l}$ and $a_{l}$ are the spline coefficients. A detailed description of the discretization procedure can be found in Refs. $[3-5,18,19]$. We apply the so-called phase factor transform [18] to all perturbed quantities in the code. The integrals over the gyroangle are approximated with an N-point discrete sum [19-21]. The cancellation problem $[1,3]$, which in the mixed-variable formulation is related only to the correction $\delta A_{\|}$of the parallel magnetic potential [see Eq. (15)], is solved using the iterative scheme introduced in Refs. [2, 5].

We start with the case described in Ref. [8] used for the International Tokamak Physics Activity (ITPA) benchmark [22]: a large-aspect-ratio tokamak with a circular cross-section, the minor radius $r_{\mathrm{a}}=1 \mathrm{~m}$, the major radius $R_{0}=10 \mathrm{~m}$, the magnetic field on axis $B_{0}=3 \mathrm{~T}$, and the safety factor profile $q(r)=1.71+0.16\left(r / r_{\mathrm{a}}\right)^{2}$ (here, $r$ is the small radius). The background plasma profiles (Maxwellian unperturbed distribution function) are chosen to be flat with the ion (Hydrogen) density $n_{i}=2 \times 10^{19} \mathrm{~m}^{-3}$, and flat temperatures $T_{i}=T_{e}=1 \mathrm{keV}$. Following further Ref. [8], we choose a Maxwellian for the unperturbed distribution function of the fast particles (deuterium ions), a flat fast-particle temperature $T_{f}$ and the fast particle density given by the expression:

$$
n_{f}(s)=n_{0 f} \exp \left[-\frac{\Delta_{\mathrm{n} f}}{L_{\mathrm{n} f}} \tanh \left(\frac{s-s_{\mathrm{n} f}}{\Delta_{\mathrm{n} f}}\right)\right]
$$

with $s_{\mathrm{n} f}=0.5$ being the position of the maximal value of $\kappa_{\mathrm{n} f}=\left|\nabla n_{f}\right| / n_{f}$. Further, $n_{0 f}=0.75 \times 10^{17} \mathrm{~m}^{-3}$ is the fast particle density at $s=s_{\mathrm{n} f}, \Delta_{\mathrm{n} f}=0.2$ the "width" of the density profile, and $L_{\mathrm{n} f}=0.3$ the "length" of the fast-particle density profile (this parameter determines how large the density gradient is). For further details, see Refs. [8, 22]. We also note that the ITPA benchmark parameters are optimised for the code verification purposes; they do not correspond to any really existing tokamak.

We consider a Toroidal Alfvén Eigenmode (TAE) with the toroidal mode number $n=-6$ and the dominant poloidal harmonics $m=10$ and $m=11$. The ideal MHD frequency of this mode $\omega_{\mathrm{MHD}}=0.414 \times 10^{6} \mathrm{rad} / \mathrm{s}$. In Figs. 1 and 2 , the gyrokinetic frequency and the gyrokinetic growth rate of the TAE mode are plotted as functions of the fast-ion temperature. Here, we compare the simulations performed using the usual $p_{\|}$-formulation with the new mixed-variable simulations introduced in this paper.

One sees that the agreement between the two schemes is very good. Interestingly, the 
numerical requirements are much more relaxed in the mixed-variable case, where the minimum time step needed is an order of magnitude larger than the time step required by the $p_{\|}$-formulation. Here, one could hypothesise that the cancellation problem couples to eventual inaccuracies in the time-integration scheme, strongly amplifying them as a consequence.

The next case to be considered is a similar TAE mode in the same magnetic geometry, but now with a smaller perpendicular mode number $k_{\perp}$ corresponding to the toroidal mode number $n=-2$ and the dominant poloidal harmonics $m=3$ and $m=4$. Because of the cancellation problem (which scales as $1 / k_{\perp}^{2}$, see Ref. [3]), this case is much more complicated than the previous one. When attempted with the conventional $p_{\|}$-formulation and using the time step $\omega_{\mathrm{ci}} \Delta t=5.0$, the simulations end in a severe numerical instability within a few time steps (see the mode structure in Fig. 3 for $\omega_{\text {ci }} t=25$, i. e. at the fifth time step). The simulation could be carried out for a longer time, but an order of magnitude smaller time step $\left(\omega_{\mathrm{c} i} \Delta t=0.5\right)$ had to be implemented. Of course, the computational cost of such simulations increases considerably. Despite the considerable computational effort, the numerical instability continues to develop inevitably also in this case, though at later times. The resulting radial pattern and the evolution of the electrostatic potential are shown in Figs. 4 and 5.

In contrast, the mixed-variable simulations can be performed successfully even at the small values of $k_{\perp}$, without any substantial numerical complications and at much larger time steps $\omega_{\mathrm{c} i} \Delta t=5.0$. The resulting mode structure is shown in Fig. 6. As expected, one observes a typical TAE pattern. In Fig. 7, the mode evolution is plotted at different flux surfaces (here $s$ denotes the toroidal flux). One sees that a coherent unstable mode develops. The frequency of this mode $\omega=3.95 \times 10^{5} \mathrm{rad} / \mathrm{s}$ aligns well with the TAE gap, as expected. The growth rate $\gamma=2.68 \times 10^{4} \mathrm{rad} / \mathrm{s}$ is caused by the fast-ion destabilisation mechanism.

\section{CONCLUSIONS}

In this paper, we have proposed a novel method to greatly mitigate the cancellation problem in electromagnetic gyrokinetic simulations with an appropriate choice of gyrokinetic variables. This choice is based on a particular form of parallel Ohm's law. As an example, the ideal version has been used throughout this paper. The scheme has been validated with a gyrokinetic PIC code using an example of the fast-ion driven TAE instability (the ITPA 
benchmark case [22]). Furthermore, it has been shown that electromagnetic gyrokinetic simulations at small perpendicular mode numbers $k_{\perp}$ are feasible with the new method, despite the cancellation problem scaling as $1 / k_{\perp}^{2}$ (see Ref. [3]). Notably, our scheme is not limited to the PIC approach, which has been used here merely as a validation framework.

The implementation of an appropriate version of Ohm's law is important for the scheme to work, in the sense that it should give a good approximation for the actual physical $E_{\|}$ to be simulated. For example, ideal Ohm's law $E_{\|}=0$, considered here, seems to be a good approximation for the shear Alfvénic perturbations such as the TAE, but would be less accurate for electromagnetic drift modes. Clearly, it is straightforward to extend the scheme used here to other, more advanced, forms of parallel Ohm's law, taken e.g. from gyrofluid equations. Such extensions will be considered in future work.

\section{ACKNOWLEDGEMENTS}

We acknowledge the support of Per Helander for this work. We thank Jürgen Nührenberg for carefully reading this manuscript. Discussions on the cancellation problem during the workshop on "Modelling kinetic aspects of Global MHD Modes" organised by Egbert Westerhof at the Lorentz Center in Leiden were very helpful. In particular, remarks by Arthur Peeters are appreciated. This work was carried out using the HELIOS supercomputer system at Computational Simulation Centre of International Fusion Energy Research Centre (IFERC-CSC), Aomori, Japan, under the Broader Approach collaboration between Euratom and Japan, implemented by Fusion for Energy and JAEA. Also, some simulations have been performed on the local cluster in Greifswald (support of Henry Leyh is appreciated). The project has received funding from the Euratom research and training program 2014-2018.

\section{APPENDIX A: DIRECT VARIABLE TRANSFORMATION}

In the appendix, we attempt to derive the mixed-variable equations of motion using the direct variable transformation approach. A simple way to do it seems to be the following. 
1. Take the standard gyrokinetic equations [13] solved by majority of the codes:

$$
\begin{aligned}
\dot{\mathbf{R}} & =v_{\|} \mathbf{b}^{*}+\frac{1}{q B} \mathbf{b} \times \mu \nabla B+\frac{\mathbf{b}}{B} \times \nabla\left\langle\phi-v_{\|} A_{\|}\right\rangle-\frac{q}{m}\left\langle A_{\|}\right\rangle \mathbf{b}^{*} \\
\dot{v}_{\|} & =-\frac{\mu}{m} \mathbf{b}^{*} \cdot \nabla B-\frac{q}{m} \mathbf{b}^{*} \cdot \nabla\left\langle\phi-v_{\|} A_{\|}\right\rangle \\
\mathbf{b}^{*} & =\mathbf{b}+\frac{m v_{\|}}{q B}(\nabla \times \mathbf{b})_{\perp}
\end{aligned}
$$

2. Split the parallel magnetic potential into the 'MHD part' and the residual part:

$$
A_{\|}=A_{\|}^{(\mathrm{MHD})}+\delta A_{\|}
$$

3. Transform the 'parallel velocity' variable:

$$
v_{\|}=v_{\|}^{(\mathrm{m})}+\frac{q}{m}\left\langle A_{\|}^{(\mathrm{MHD})}\right\rangle
$$

4. Substitute the new 'parallel velocity' into the equations of motion and obtain, to the first order, the following:

$$
\begin{aligned}
& \dot{\mathbf{R}}=v_{\|}^{(\mathrm{m})} \mathbf{b}_{(\mathrm{m})}^{*}+v_{\|}^{(\mathrm{m})} \frac{\left\langle A_{\|}^{(\mathrm{MHD})}\right\rangle}{B}(\nabla \times \mathbf{b})_{\perp} \cdot \mathbf{b}_{(\mathrm{m})}^{*}+\frac{1}{q B} \mathbf{b} \times \mu \nabla B+ \\
& +\frac{\mathbf{b}}{B} \times \nabla\left\langle\phi-v_{\|}^{(\mathrm{m})} A_{\|}\right\rangle-\frac{q}{m}\left\langle\delta A_{\|}\right\rangle \mathbf{b}_{(\mathrm{m})}^{*} \\
& \dot{v}_{\|}^{(\mathrm{m})}+\frac{q}{m} \frac{\partial}{\partial t}\left\langle A_{\|}^{(\mathrm{MHD})}\right\rangle=-\frac{\mu}{m} \mathbf{b}_{(\mathrm{m})}^{*} \cdot \nabla B-\frac{\mu}{m} \frac{\left\langle A_{\|}^{(\mathrm{MHD})}\right\rangle}{B}(\nabla \times \mathbf{b})_{\perp} \cdot \nabla B- \\
& \quad-\frac{q}{m} \mathbf{b}_{(\mathrm{m})}^{*} \cdot \nabla\left\langle\phi-v_{\|}^{(\mathrm{m})} A_{\|}\right\rangle \\
& \mathbf{b}_{(\mathrm{m})}^{*}=\mathbf{b}+\frac{m v_{\|}^{(\mathrm{m})}}{q B}(\nabla \times \mathbf{b})_{\perp}
\end{aligned}
$$

5. Substitute the ideal Ohm's law, Eq. (5), and arrive at the equations of motion, written to the first order:

$$
\begin{aligned}
\dot{\mathbf{R}}= & v_{\|}^{(\mathrm{m})} \mathbf{b}_{(\mathrm{m})}^{*}+v_{\|}^{(\mathrm{m})} \frac{\left\langle A_{\|}^{(\mathrm{MHD})}\right\rangle}{B}(\nabla \times \mathbf{b})_{\perp} \cdot \mathbf{b}_{(\mathrm{m})}^{*}+\frac{1}{q B} \mathbf{b} \times \mu \nabla B+ \\
& +\frac{\mathbf{b}}{B} \times \nabla\left\langle\phi-v_{\|}^{(\mathrm{m})} A_{\|}\right\rangle-\frac{q}{m}\left\langle\delta A_{\|}\right\rangle \mathbf{b}_{(\mathrm{m})}^{*} \\
\dot{v}_{\|}^{(\mathrm{m})}= & -\frac{\mu}{m} \mathbf{b}_{(\mathrm{m})}^{*} \cdot \nabla B-\frac{\mu}{m} \frac{\left\langle A_{\|}^{(\mathrm{MHD})}\right\rangle}{B}(\nabla \times \mathbf{b})_{\perp} \cdot \nabla B- \\
& -\left[\frac{v_{\|}^{(\mathrm{m})}}{B}(\nabla \times \mathbf{b})_{\perp} \cdot \nabla \phi-\frac{q}{m} v_{\|}^{(\mathrm{m})} \mathbf{b}_{(\mathrm{m})}^{*} \cdot \nabla\left\langle A_{\|}\right\rangle\right]
\end{aligned}
$$


Neither Eq. (A9) nor Eq. (A10) coincide with the corresponding Eqs. (8) and (9) in the main text, respectively. This indicates that the simple procedure, described above, does not suffice. One needs a more extensive calculation which would take into account all other subtleties of the direct variable transformation from the $p_{\|}$-formulation to the mixedvariable formulation, such as the differences in other variables and distribution functions. All these subtleties are accounted for automatically by the Lie transform approach. A complete derivation of the mixed-variable equations using the direct variable transformation approach is beyond the scope of the present paper and can be done in future.

[1] Y. Chen and S. Parker, Phys. Plasmas 8, 2095 (2001).

[2] Y. Chen and S. Parker, J. Comp. Phys 189, 463 (2003).

[3] A. Mishchenko, R. Hatzky, and A. Könies, Phys. Plasmas 11, 5480 (2004).

[4] A. Mishchenko, A. Könies, and R. Hatzky, in Proc. of the Joint Varenna-Lausanne International Workshop (Società Italiana di Fisica, Bologna, 2004).

[5] R. Hatzky, A. Könies, and A. Mishchenko, J. Comp. Phys. 225, 568 (2007).

[6] J. Candy and R. E. Waltz, J. Comp. Phys. 186, 545 (2003).

[7] A. Mishchenko, R. Hatzky, and A. Könies, Phys. Plasmas 15, 112106 (2008).

[8] A. Mishchenko, A. Könies, and R. Hatzky, Phys. Plasmas 16, 082105 (2009).

[9] A. Mishchenko, A. Könies, and R. Hatzky, Phys. Plasmas 18, 012504 (2011).

[10] A. Mishchenko and A. Zocco, Phys. Plasmas 19, 122104 (2012).

[11] Y. Chen and S. Parker, Phys. Plasmas 8, 441 (2001).

[12] Z. Lin and L. Chen, Phys. Plasmas 8, 1447 (2001).

[13] T. S. Hahm, W. W. Lee, and A. J. Brizard, Phys. Fluids 31, 1940 (1988).

[14] V. Kornilov, R. Kleiber, R. Hatzky, L. Villard, and G. Jost, Phys. Plasmas 11, 3196 (2004).

[15] A. J. Brizard and T. S. Hahm, Reviews of Modern Physics 79, 421 (2007).

[16] C. de Boor, A Practical Guide to Splines (Springer-Verlag, New York, 1978).

[17] K. Höllig, Finite Element Methods with B-Splines (Society for Industrial and Applied Mathematics, Philadelphia, 2003).

[18] M. Fivaz, S. Brunner, G. de Ridder, O. Sauter, T. M. Tran, J. Vaclavik, L. Villard, and K. Appert, Comp. Phys. Commun. 111, 27 (1998). 
[19] A. Mishchenko, A. Könies, and R. Hatzky, Phys. Plasmas 12, 062305 (2005).

[20] W. W. Lee, J. Comp. Phys. 72, 243 (1987).

[21] R. Hatzky, T. M. Tran, A. Könies, R. Kleiber, and S. J. Allfrey, Phys. Plasmas 9, 898 (2002).

[22] A. Könies, S. Briguglio, N. Gorelenkov, T. Fehér, M. Isaev, P. Lauber, A. Mishchenko, D. A. Spong, Y. Todo, W. A. Cooper, R. Hatzky, R. Kleiber, M. Borchardt, G. Vlad, ITPA EP TG, in Proc. of 24th Fusion Energy Conf. (IAEA, Vienna, 2012). 


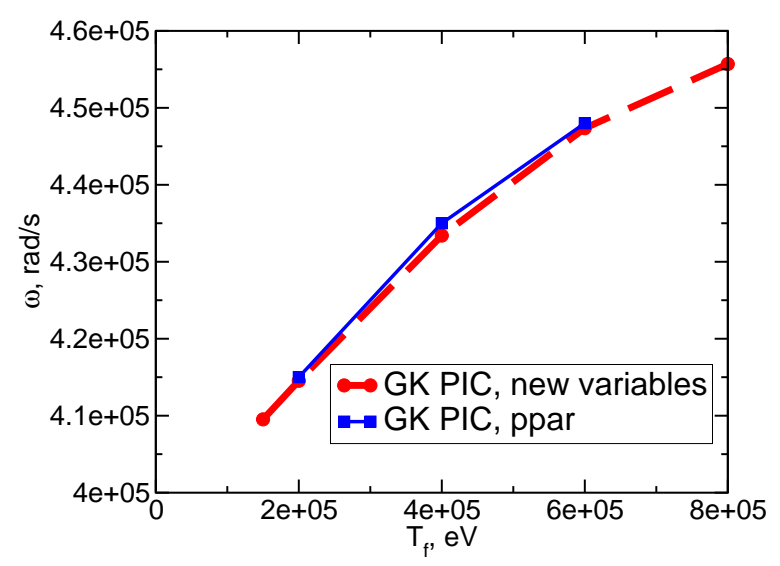

FIG. 1: (Color online) The TAE frequency in the ITPA benchmark [22]. Gyrokinetic simulations in $p_{\|}$-variables are compared with the gyrokinetic simulations in mixed variables. 


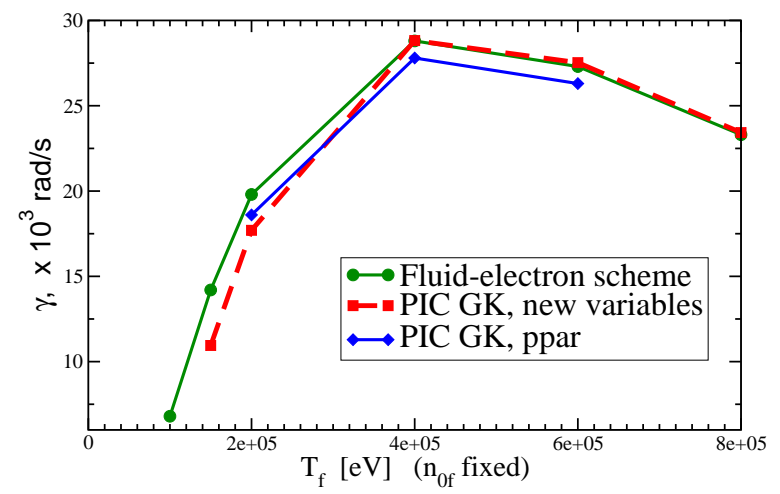

FIG. 2: (Color online) The TAE growth rate in the ITPA benchmark [22]. Gyrokinetic simulations in $p_{\|}$-variables are compared with the gyrokinetic simulations in mixed variables and with simulations using the fluid-electron scheme similar to Refs. [11, 12]. 


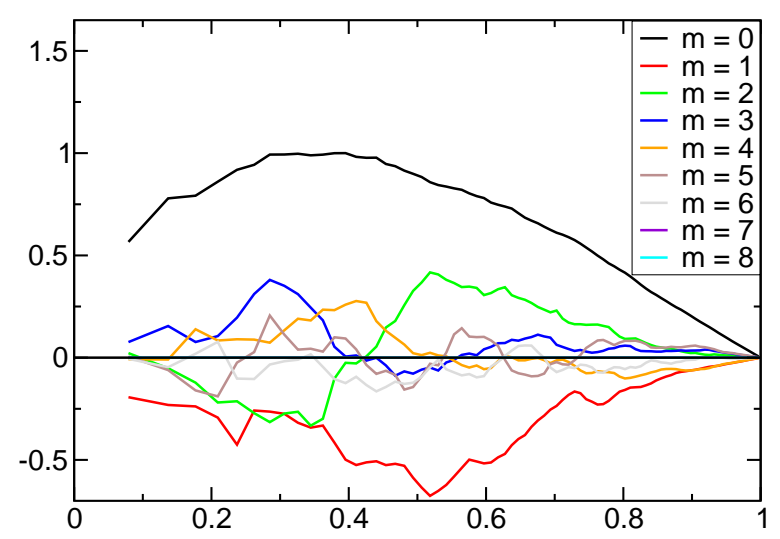

FIG. 3: (Color online) Electrostatic potential mode structure obtained using the $p_{\|}$-formulation and the time step $\omega_{\mathrm{c} i} \Delta t=5.0$. A severe numerical instability produces an erratic structure of the electrostatic potential within few time steps. Here, the fifth time step is shown. The dominant harmonic has the poloidal mode number $m=0$ and corresponds to the smallest $k_{\perp}$ in the Fourier filter implemented (i. e. the mode is non-physical). The case, attempted here, corresponds to the TAE mode with the toroidal mode number $n=-2$ and dominant poloidal mode numbers $m=3$ and $m=4$. Other parameters (magnetic geometry etc.) coincide with the ITPA benchmark. The fast-ion temperature is $T_{f}=0.4 \mathrm{MeV}$. 


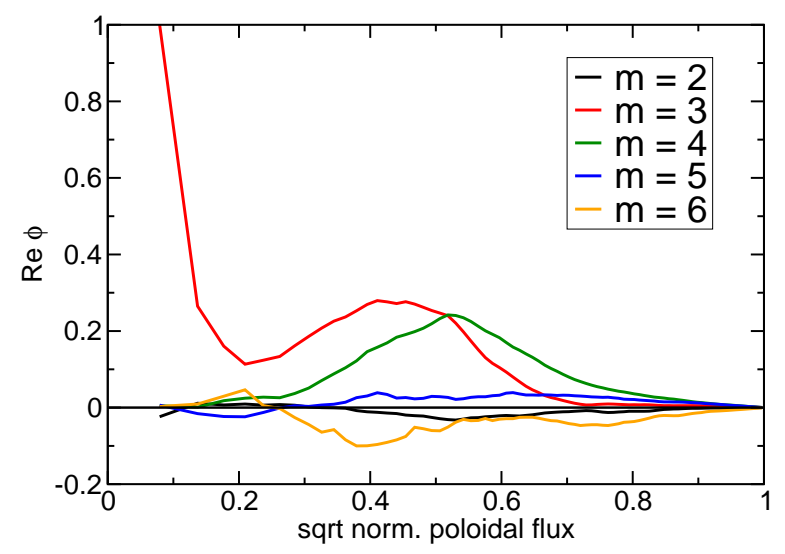

FIG. 4: (Color online) Electrostatic potential mode structure obtained using the $p_{\|}$-formulation. One sees that a numerical instability develops with a characteristic "singularity" at the magnetic axis. The time step $\omega_{\mathrm{c} i} \Delta t=0.5$ has been used here. All other parameters as in Fig. 3. 


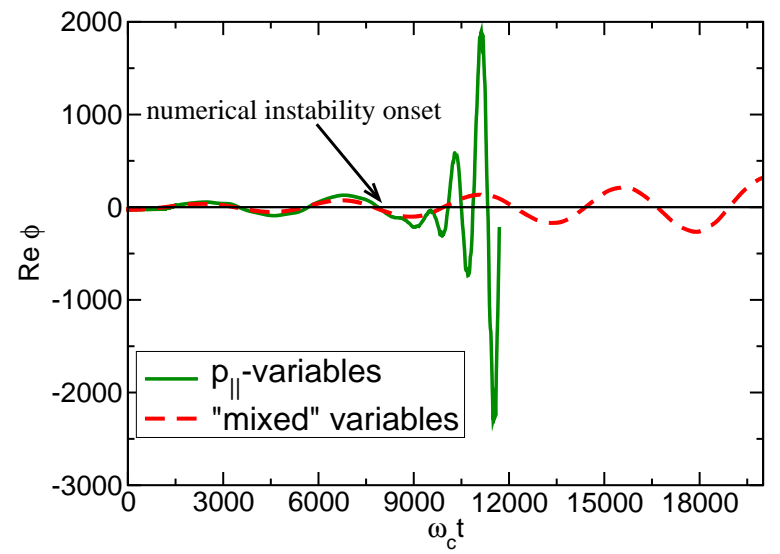

FIG. 5: (Color online) Evolution of the electrostatic potential at $s=0.3$ (here $s$ denotes the toroidal flux). The simulation using the $p_{\|}$-formulation is compared with the mixed-variable simulation. A numerical instability sets on at the time $\omega_{\mathrm{c} i} t \approx 9 \times 10^{3}$. The time step $\omega_{\mathrm{c} i} \Delta t=0.5$ has been used in the simulation employing the $p_{\|}$-formulation. All other parameters as in Fig. 3. 


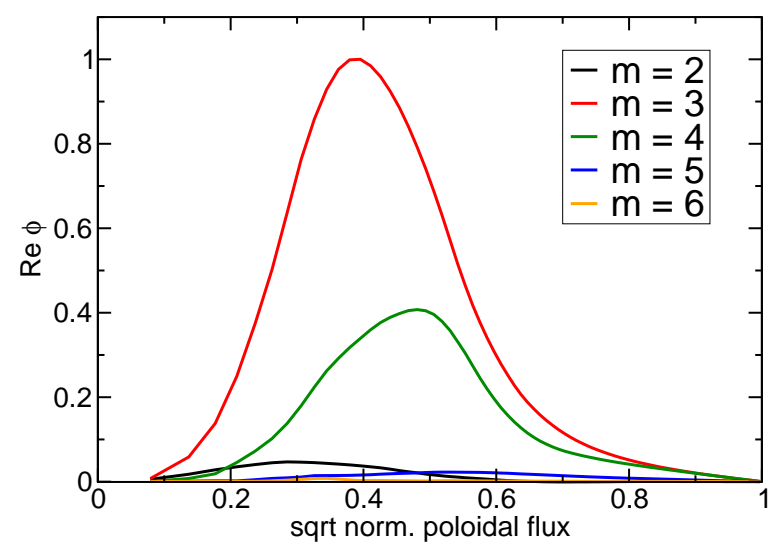

FIG. 6: (Color online) Electrostatic potential mode structure obtained in the mixed-variable simulations. One sees that a clear TAE mode structure, with the toroidal mode number $n=-2$ and the dominant poloidal mode numbers $m=3$ and $m=4$, appears in the simulation. All parameters (including the time step $\omega_{\mathrm{c} i} \Delta t=5.0$ ) are as in Fig. 3. The striking difference compared to Fig. 3 is solely due to the 'mixed variables' used here instead of the $p_{\|}$-formulation. 


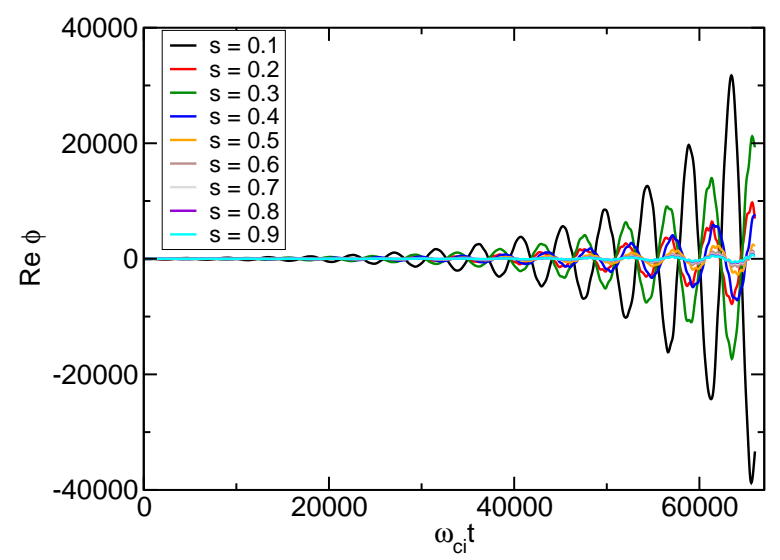

FIG. 7: (Color online) Evolution of the electrostatic potential at different flux surfaces (here $s$ denotes the toroidal flux). One sees that a coherent, physically unstable mode develops. The frequency of the mode is in the TAE gap. All parameters (including the time step $\omega_{\mathrm{c} i} \Delta t=5.0$ ) are as in Fig. 3. The mode evolves without any sign of a numerical instability for times much longer than in Fig. 5 although the time step used here is an order of magnitude larger. The only difference are the 'mixed variables' used here instead of the $p_{\|}$-formulation. 